\title{
The Challenges of Managing Developmental Disorders During a Pandemic
}

\author{
Morgan T. Sammons \\ Published online: 29 July 2020 \\ (C) National Register of Health Service Psychologists 2020
}

How do we treat common psychological disorders during a pandemic? Although many mental health providers have shifted to online service provision, it is clear that there are inherent limitations to internet-based psychotherapy. Not only is patient engagement made more challenging, the applicability of therapeutic interventions to real-world situations is complicated by the reduced availability of social interactions. Many social interactions now take place online, where standards for interpersonal conduct may differ significantly from face-to-face interactions. Numerous previous studies have demonstrated roughly equivalent outcomes for internetbased versus in-person psychotherapy, but none that I am aware of have done so in the context of a pandemic, where not just therapy but most social activities are largely restricted to the electronic environment and the applicability of therapeutic interventions is difficult to assess. Published studies of online psychotherapies usually include methods for adapting psychotherapeutic techniques, but few if any include strategies for substituting electronic interactions for those present in 'real world' school, work, and social activities.

Developmental disorders in particular may be challenging to address in a world where social interaction is limited. Lack of corrective peer feedback may lead adolescent girls to negatively evaluate their sense of self. Lack of external constraints and peer support may prevent teenagers with chronic illnesses such as diabetes to conform with behaviors requisite for effective disease management. Dysfunctional interactions with parents and caregivers, up to and including abusive behaviors, may be magnified when usual social outlets are not present. Paradoxically, social anxiety may be exaggerated, not reduced, in online interactions where normal nonverbal cues and disengagement strategies are absent. Isolation may be a risk factor for adolescents with suicidal behavior, eating disorders, or self-injurious behavior. As we recognize, absence of the agora is not in itself a sufficient intervention for agoraphobia, manifested as it is by numerous dysfunctional behaviors affecting self and others. Many of the articles in this issue of the Journal of Health Service Psychology deal to some extent with developmental disorders. Challenges to their management in a virtual environment, but more conspicuously the absence of routine interpersonal interactions will be apparent to any reader.

Adolescent experimentation with healthy risk-taking and boundary setting is complicated during a pandemic. Not only are adolescent norms regarding self-image and independence challenged by restrictions required to maintain physical health, critical aspects of socialization are absent with the cessation of in-person school, sport, and other activities. Weissbrod and Colangelo (2020) outline the centrality of healthy risk-taking in the development of self-acceptance and social confidence. Such behavior requires interpersonal interactions - and challenges - in order for adolescents to develop a healthy sense of self. Given that much socialization now takes place in an electronic environment, where opportunities for negative interactions are unfettered by the norms affecting face-to-face communication, girls who are prone to automatic negative biases and anxiety may be more susceptible to the often toxic environment that pervades social media. Self-limiting participation in social media may not be perceived as an option by adolescents who are deprived of engagement in school, sport, and extracurricular social activities. The authors note that traditional cognitive-behavioral approaches may be insufficient when working with socially anxious girls. Exercises enhancing self-compassion, mindfulness, and the ability to engage in healthy risk-taking are key aspects of successful treatment, which must be adapted to an environment that is largely devoid of traditional interpersonal interaction and support.

Recovery from physiological injury is highly variable, as Aylward (2020) notes in his article detailing the importance of assessment —often serial assessment—in children with birthrelated insult or early developmental trauma. Results of such assessments are subject to interrater variability, and point in time testing may have significant consequences, such as 
eligibility for early childhood services. Severity of physical injury does not predict outcome, as in the case of intraventricular hemorrhage described in the article. The literature regarding the sequelae of mild intraventricular hemorrhage remains unsettled, increasing the importance of neuropsychological assessment, but as Aylward reminds us, testing without a specific purpose or intervention as a goal is not a productive use of the clinician's or family's time.

Adolescent diabetes is a particular challenge for patients, families, and clinicians. The normal and often turbulent process of adolescent maturation and individuation is complicated by the knowledge that a life-threatening condition must be scrupulously managed for optimal health. The prevalence of type 2 diabetes in youth has increased substantially over time. This is associated not only with deleterious dietary habits, weight gain, and more sedentary lifestyles but type 2 diabetes associated with the administration of psychotropic medications is an increasingly common cause. Caccavale and Monaghan (2020) note that in addition to comorbid anxiety and depression, diabetes distress is a recognizable clinical syndrome in as many as one third of adolescents with type 1 or type 2 diabetes. Regardless of the nature of the psychological overlay, such conditions demonstrably affect both the ability to effectively manage the condition successfully and the attainment of age-appropriate social and developmental milestones. A treatment approach that is focused on behavioral change, not the pursuit of overall goals such as reduced A1C levels, provides the best means of achieving adaptation, but addressing features associated with diabetes distress (distress associated with the emotional, physical, interpersonal and patient-role components of diabetes management) is requisite for successful intervention.

Nonspecificity in diagnosis and treatment is also the hallmark of adult attention deficit disorder, and as Staley Shumaker and Hoyt (2020) outline, a standardized process of testing and clinical evaluation can enhance diagnostic certainty. Adult ADHD is in many respects an archetype of nonspecificity and reflects an increasingly common conundrum in mental health treatment, which I have chosen to call the "DSM fallacy": It is fallacious to believe that simply because we can describe various disorders with increasing degrees of specificity that our treatments have become correspondingly specific. While our diagnostic criteria for adult onset ADHD, a disorder that has only recently entered the nomenclature, have become more specific, our treatments are anything but. Stimulant medication combined with a behavioral regimen constitute the best evidence-supported treatment for childhood ADHD, and it is logical to presume that such a regimen will be useful in the adult variant of the disorder. Before prescribing either with confidence, we should verify that other factors do not explain the symptom presentation. Prescribers and patients alike should be aware that stimulant medications are a highly nonspecific intervention. Adults without ADHD are likely to display increases in attention and concentration if given modest doses of stimulant medication. Though generally benign in most adults who are free of underlying cardiovascular conditions, seizure disorders, eating disorders and the like, stimulant medications are recognized drugs of abuse and it behooves the provider to not only engage in systematic assessment, including psychological testing (as imperfect and imprecise as it may be) and comprehensive patient education before embarking on this course of treatment.

As with depression, the experience of chronic pain is highly idiosyncratic. Individual differences in pain expression and pain response dramatically reduce the applicability of any one approach to its management, regardless of the modality. With pharmacology, there is no single preferred approach. Decades of clinical research and experience inform us that the strength of the evidence for non-opioid medications in chronic pain remains uncertain. Opioids come with the risk of habituation and misuse, but for too long our clinical interventions have been guided by fear of these consequences rather than by the patient's clinical presentation. A recalculation of this approach to one less focused on provider risk management and more on individual patient needs is well overdue. But the limitations to any physical intervention, whether it be prescribed agents, herbals such as cannabis, or surgery, mandate consideration of some form of behavioral or psychological therapy. As Ashton-James and Ziadni (2020) note, nonspecific psychological interventions such as cognitive behavioral therapy or acceptance-based interventions do not address important psychosocial contributors to chronic pain and may indeed contribute to prolonging high-impact pain. Emotional Awareness and Expression Therapy, which has been demonstrated to be effective in single session interventions, may provide an avenue to more completely address the psychosocial factors prolonging experienced pain, at least in a self-selected group of patients. Like treatments for any other protean condition, no single intervention will be sufficient, but it promises to add another tool to the treatment armamentarium.

In sum, the pandemic presents us with both risks and opportunities. We have demonstrated with relative certainty that many psychological interventions can be adapted for online administration. Technological advances and accumulated experience over the past three decades have given us confidence that much of what we do retains its potency, even when delivered remotely. Of course, many interventions have not been tested in virtual environments, and that work remains. But perhaps the more interesting question is how patients apply these interventions in a world where traditional social interactions have been paused.

\section{References}

Ashton-James, C. E. \& Ziadni, M. S. (2020). Uncovering and Resolving Social Conflicts Contributing to Chronic Pain: Emotional 
Awareness and Expression Therapy. Journal of Health Service Psychology, 46(3). https://doi.org/10.1007/s42843-020-00017-y

Aylward, G. P. (2020). Conducting a Developmental Assessment in Young Children. Journal of Health Service Psychology, 46(3). https://doi.org/10.1007/s42843-020-00015-0

Caccavale, L. J. \& Monaghan, M. (2020). Behavioral Interventions for Youth with Diabetes. Journal of Health Service Psychology, 46(3). https://doi.org/10.1007/s42843-020-00014-1

Staley Shumaker, B. E. \& Hoyt, T. (2020). A Place for Psychological Testing in the Assessment of Adult ADHD. Journal of Health Service Psychology, 46(3). https://doi.org/10.1007/s42843-02000016-z
Weissbrod, C. S. \& Colangelo, A. (2020). Understanding and Treating Social Anxiety in Female Adolescents. Journal of Health Service Psychology, 46(3). https://doi.org/10.1007/s42843-020-00008-z

Publisher's Note Springer Nature remains neutral with regard to jurisdictional claims in published maps and institutional affiliations.

Morgan T. Sammons, PhD, ABPP, is the Executive Officer of the National Register of Health Service Psychologists, and the Editor of the Journal of Health Service Psychology. He is a retired Navy captain and was formerly the U.S. Navy's specialty leader for clinical psychology. 